\title{
A COMPARISON OF METHODS FOR MEASUREMENT OF PRESSURE IN HYDRAULIC LINES
}

\author{
Susan Sprague and Andrew Chorney \\ Naval Air Warfare Center Aircraft Division
}

\begin{abstract}
This presentation summarizes a study characterizing strain gages and pressure transducers used to measure the fluid pressure within aircraft hydraulic lines. A series of laboratory calibrations and finite element analyses was performed to demonstrate the quality of data from both pressure transducers and strain gages under variations in both temperature and external strains on the hydraulic lines. Strain gages showed a marked susceptibility to external strains on hydraulic lines, and wide variations in susceptibility to temperature changes. Pressure transducers were found to be relatively immune to both conditions. It is recommended that strain gages be used for trend data only.
\end{abstract}

\section{KEY WORDS}

Strain gages, pressure transducers, hydraulic lines, measurement uncertainty, and aircraft.

\section{INTRODUCTION}

Measurement of a parameter can often be achieved using a variety of methods. The choice of methodology is made according to the requirements of the application. Considerations include the type of measurement; transducer specificity; electrical, physical, and mechanical characteristics; data requirements; and program constraints. In most cases the choice of transducer involves a trade-off. For example, the most accurate transducer may be too large, too heavy, or too expensive to be suitable for the application.

In 1996 an aircraft at NAWCAD was instrumented for measurement of fluid pressure within several hydraulic lines. Two methodologies were used to measure the pressure: externally placed strain gages to measure the hoop stress on the lines exerted by the hydraulic fluid within the lines (indirect pressure measurement), and pressure transducers inserted within the lines to measure the pressure of the hydraulic fluid (direct pressure measurement)

The choice of methodology was based on trade-off. The highest priority in the considerations above was given to safety of flight, with data accuracy the second priority. The strain gages were determined to 
be the method of choice with respect to safety of flight and were therefore used for flight testing whenever possible. The accuracy of the gages was in question, and therefore a series of ground tests was conducted using both transducer types simultaneously for comparison of results. In all cases the transducer data was considered to be "truth data" and the strain gage data was evaluated against the truth data. The ground test results were included in the final decision process concerning which transducer type to use during flight-testing. The final configuration was a combination of transducers and strain gages that best met the requirements of the program. During the ensuing series of test flights anomalies were noted in the strain gage data including an unusual drifting characteristic. In some cases this drifting characteristic was severe enough to preclude reliable interpretation of the results.

In order to better characterize the drift characteristics and overall accuracy of the strain gages a series of post-flight-test calibrations was undertaken under varying conditions of temperature and strain on the lines.

\section{METHODOLOGY}

Since the cost and time available to perform the post calibrations was not infinite, the project engineer was consulted for determination of which lines to test. One each of the "best" and "worst" lines was chosen for characterization. The characterizations were performed on LEF2LSO (best) and LEF5LHDU (worst). Each line was subjected to calibration under a variety of combinations of temperature and strain, with the output from a transducer and the existing strain gages being measured simultaneously. Additionally, the lines were modeled using finite element analysis (FEA). The results of the calibrations were analyzed for measurement uncertainty, and used for verification of the FEA models. Once the FEA models were validated further strains were applied to the models in order to obtain additional characterization data.

Experimental setup: The experimental set-up was designed to test the response of both transducer types to temperature changes, and to test the response of the strain gages to strains on the hydraulic lines that were not due to internal pressure. Strains on the hydraulic lines were not expected to affect the conventional pressure transducers, so less emphasis was placed on subjecting these to external strains. However, when it was feasible to do so, the conventional pressure transducers were subjected to the same strains as were the strain gages.

Analysis of Measurement Uncertainty: The Abernethy Measurement Uncertainty Method was used for the uncertainty analysis. Systematic (bias) error and random (scatter) error were determined separately, then combined for an overall estimate of the $95 \%$ uncertainty ranges. The equation used to obtain the $95 \%$ uncertainty range may be found after figure 4 .

Conditions: Calibrations were run at various temperatures and strain conditions. Transducer outputs were measured at pressures of 0, 500, 1000, 1500, and 2000 psig (pounds per square inch, gage). Calibration runs were performed at temperatures of $32^{\circ} \mathrm{F}, 70^{\circ} \mathrm{F}, 100^{\circ} \mathrm{F}, 150^{\circ} \mathrm{F}$, and $200^{\circ} \mathrm{F}$, and under strain conditions of no strain (Un), compression (C), tension (T), and bending in two directions: up (Up) and down (D). 
Data Manipulation: The error sources for each post-calibration run were temperature and strain. The results of the calibrations performed on unstrained lines at $70^{\circ} \mathrm{F}$ (nominal room temperature) at each pressure level were used as the "truth" values for those pressure levels. All results were converted to \% of full scale so that the two methodologies (pressure transducer and strain gage) could be directly compared.

Finite Element Analysis: The decision to use FEA was not made until after the first series of calibrations had been run, including the compression runs on the "good" line. As a consequence of this late decision, the compression runs on this line were performed at an unknown level of compression. Unfortunately, that series of runs presented an anomaly that could not be explained: the experimental results could not be duplicated using the FEA model.

\section{RESULTS}

The results of this study indicated that the measurement of hydraulic pressure using external strain gages is considerably less accurate than when pressure transducers are used. The strain gages are highly subject to cross-reactions due to movement and stresses on the hydraulic lines. Temperature compensation on the strain gages was very good in one case (LEF2LSO), and very poor in the other (LEF5LHDU).

Overall parameter measurement uncertainty: The overall values are shown in Table 1 below. The abbreviation "XDCR" is used to indicate a pressure transducer.

\section{Table 1: Overall measurement uncertainty}

$\begin{array}{lllll}\text { Parameter } & \text { Transducer } & \text { B } & \text { S } & \text { U95 } \\ \text { LEF2LSO } & \text { Strain Gage } & 0.53 \% & 9.18 \% & 18.37 \% \\ \text { LEF2LSO } & \text { XDCR } & 0.27 \% & 1.28 \% & 2.57 \% \\ \text { LEF5LHDU } & \text { Strain Gage } & -4.05 \% & 11.00 \% & 22.37 \% \\ \text { LEF5LHDU } & \text { XDCR } & 0.04 \% & 1.06 \% & 2.11 \%\end{array}$

Most of the strain gage error was displayed as offset of the y-intercept, with very little error due to slope changes. Each individual run showed relatively little scatter. The width of the peaks in the \%Error graphs is therefore directly related to error input from the opposing error source (Force or Temperature). The primary error source for the LEF2LSO strain gage was found to be external strain. The LEF5LHDU strain gage responded to changes in both external strain and temperature. It is reasonable to expect that bias error would be introduced when the lines were installed in the aircraft. Bias error due to external strain induced at installation of the line can be zeroed out through signal conditioning. Under flight conditions drifting anomalies as the lines undergo strains caused by structural shifts, heating, vibration, and g-forces along three axes can be expected. This error can not be zeroed out through signal conditioning. 
Most of the pressure transducer error was produced at high temperature $\left(150^{\circ} \mathrm{F}\right.$ and $\left.200^{\circ} \mathrm{F}\right)$. The specifications on these transducers stated that they were compensated to $180^{\circ} \mathrm{F}$, so these results were not surprising. However, the overall uncertainty was less than $2.6 \%$ - far superior to the $18 \%$ to $22 \%$ uncertainties seen with the strain gages.

Figure 1: LEF2LSO Data by Force Type
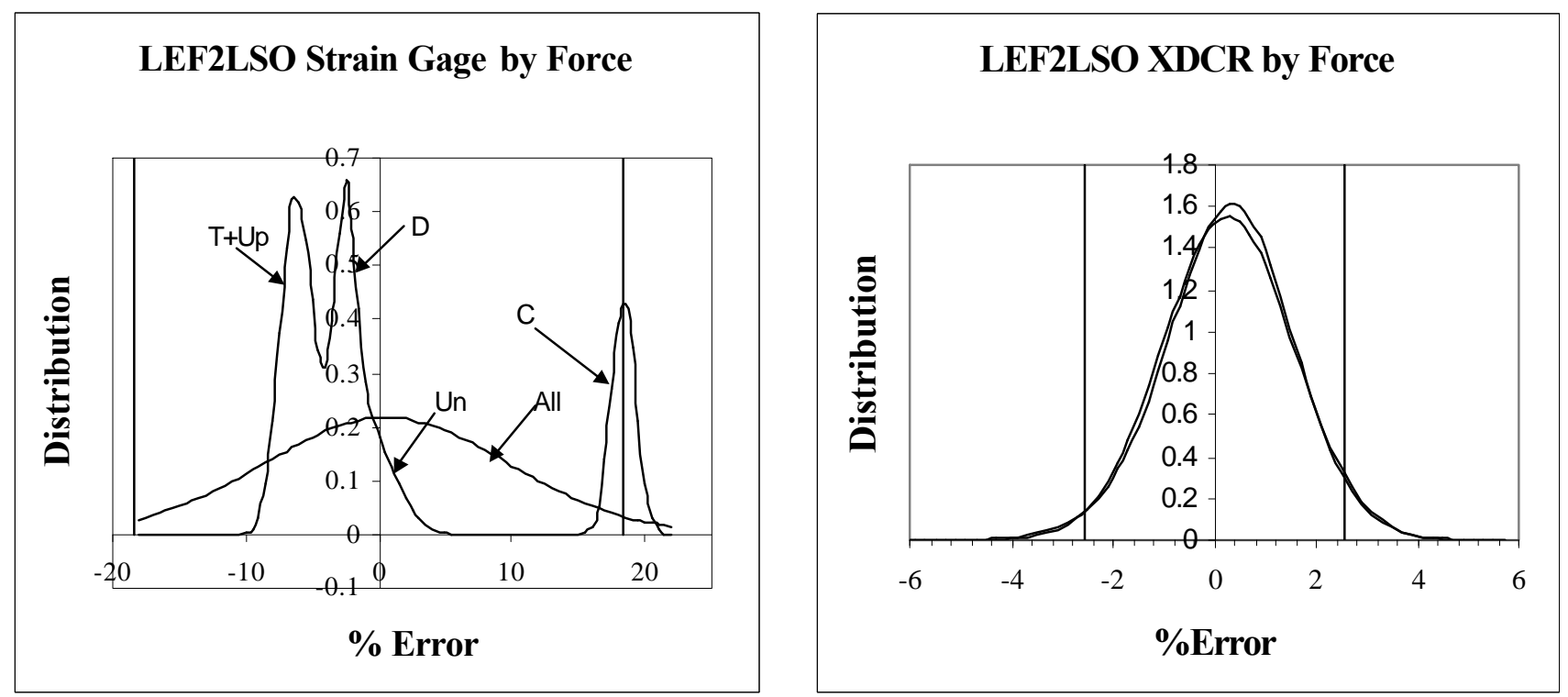

Figure 2: LEF5LHDU Data by Force Type
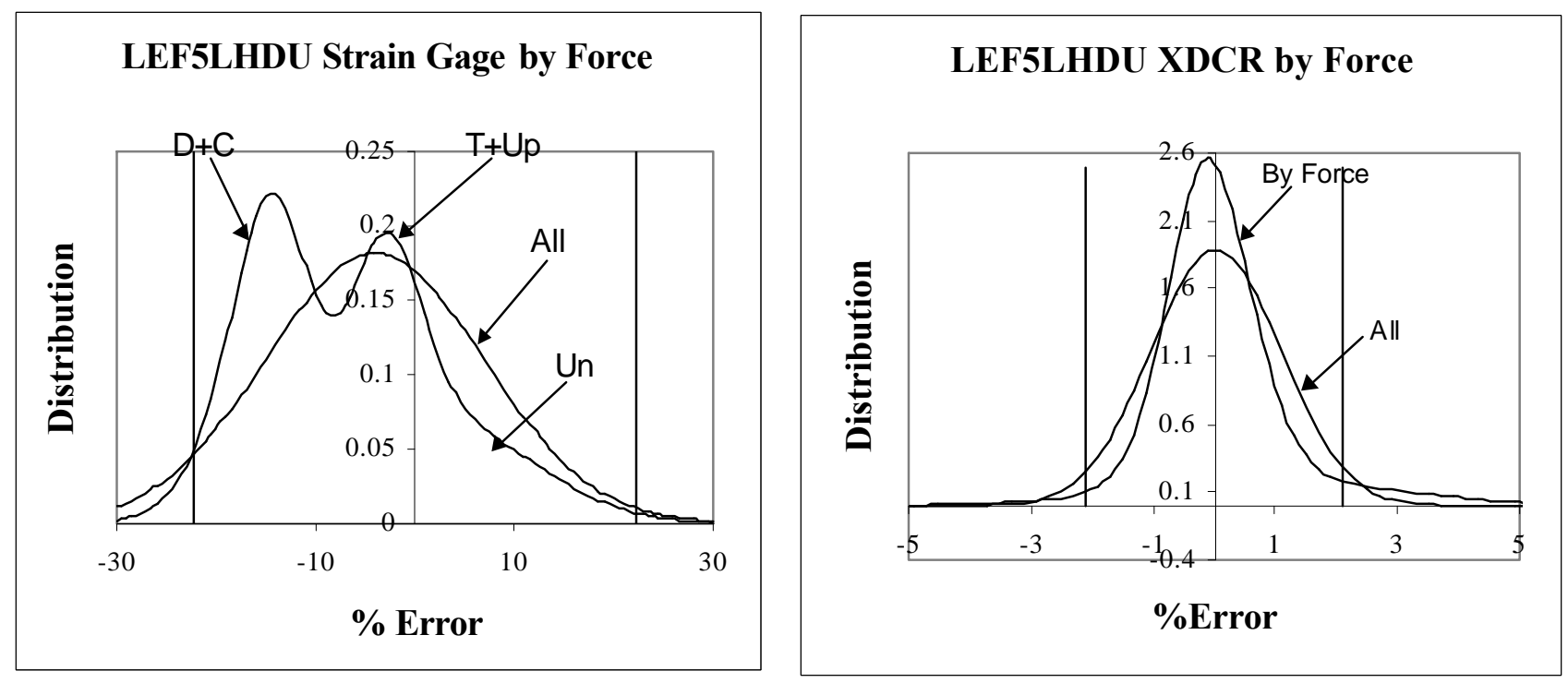
The data is illustrated below as \% Error graphs. The \% Error graphs were generated as follows: the normal distribution for each grouping was calculated in MS Excel from the bias, B (average) and scatter, $\mathrm{S}$ (standard deviation) values, and the resulting bell curves were added together point-wise along the $\mathrm{x}$ axis. This method could be used because the experimental slopes did not change significantly, only the

Figure 3: LEF2LSO Data by Temperature
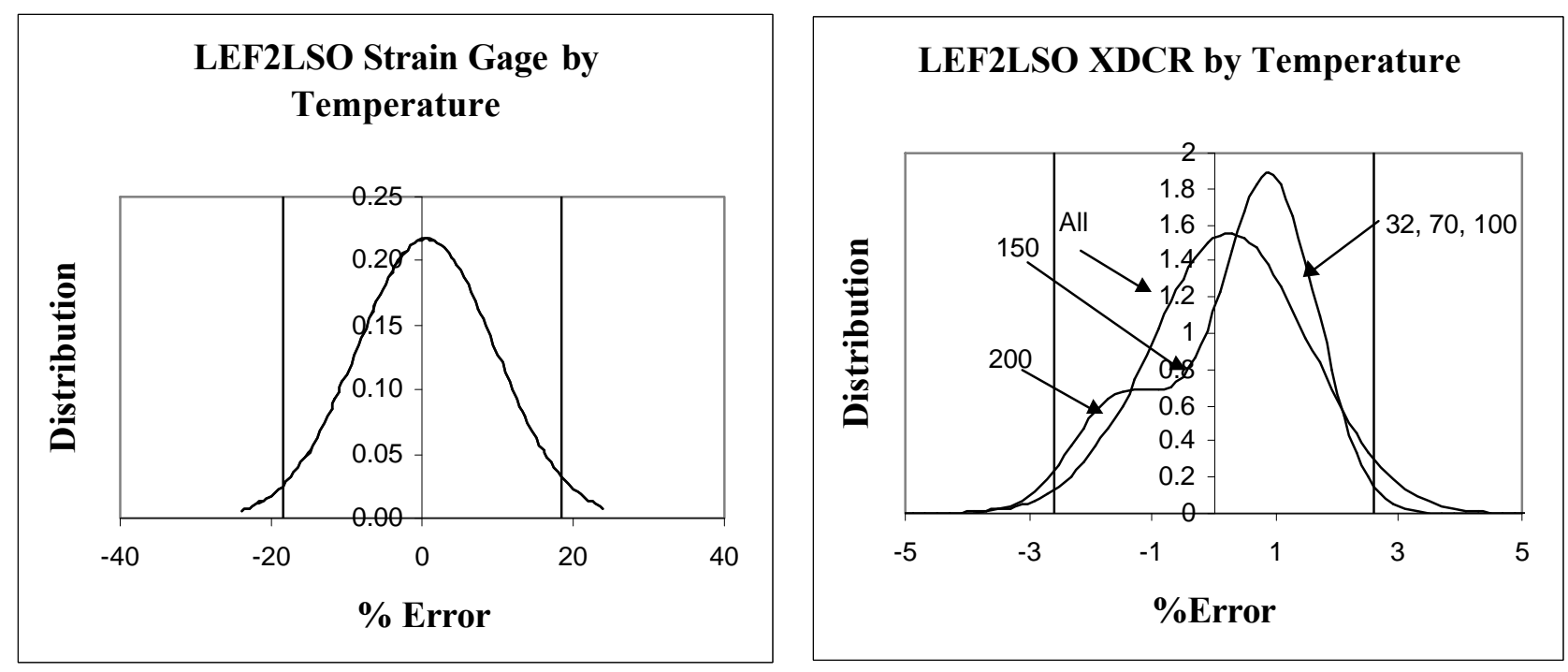

Figure 4: LEF5LHDU Data by Temperature
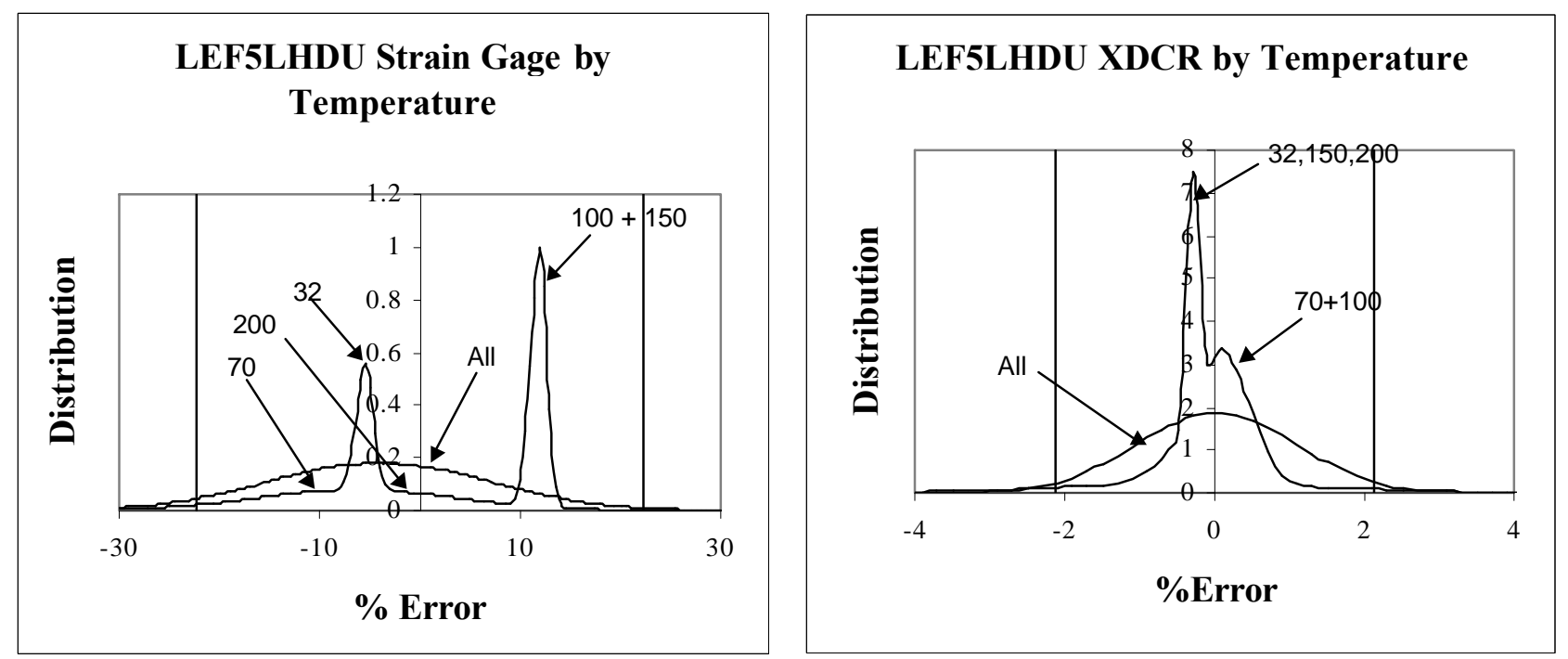

y-intercepts. The normal distribution curve for the overall (ungrouped) data was multiplied by 5 and superimposed for comparison. The overall (ungrouped) $\mathrm{U}_{95}$ values are indicated as vertical lines. The Abernethy equation is used to obtain $\mathrm{U}_{95}$ : 


$$
U_{95}= \pm \sqrt{B^{2}+(t S)^{2}}
$$

where $t$ is the Student's $t$, which approaches 2 as the number of samples approaches 30 . The positions of the peaks in the \% Error graphs correspond from left to right to y-intercept changes of corresponding point-pair least-squares-best-fit (LSBF) lines from bottom to top. The width of each peak corresponds to the point scatter that would be seen in each LSBF line.

Force Effects: Force effects are analyzed over all temperatures together. It should be noted that LEF2LSO is fully characterized, while LEF5LHDU is only partially characterized due to time and budget constraints. Calibrations were performed on LEF2LSO at all combinations of temperature and force type. Calibrations were performed on LEF5LHDU at all temperatures only in the unstrained state. Force runs were conducted only at $70^{\circ}$ and $200^{\circ}$.

LEF2LSO: The effect of external forces on the strain gage data is dramatic and obvious. It can be seen that the scatter data (seen as the bell-curve spread) is considerably lower under any one force type than when all data points (ungrouped) are considered together.

The pressure transducer, as expected, was relatively immune to external forces on the hydraulic line.

LEF5LHDU: The effect of external forces on the strain gage data is again dramatic and obvious. This time, though, there was less separation of the peaks. The probable reason will be seen in the temperature analysis.

The pressure transducer, as expected, was relatively immune to external forces on the hydraulic line.

Temperature Effects: Temperature effects are analyzed over all forces together. As was noted above, LEF2LSO is fully characterized, while LEF5LHDU is only partially characterized due to time and budget constraints. Calibrations were performed on LEF2LSO at all combinations of temperature and force type. Calibrations were performed on LEF5LHDU at all temperatures only in the unstrained state. Force runs were conducted only at $70^{\circ}$ and $200^{\circ}$.

LEF2LSO: Upon examination of the temperature effects it can be seen that for the LEF2LSO strain gage the data at all temperatures is nearly identical to the overall data. The interpretation is that the temperature compensation for this strain gage is nearly perfect, and the relatively high scatter is due solely to force effects.

The pressure transducer showed error responses to higher temperatures. This was not surprising, since this transducer was temperature compensated only to $180^{\circ}$. The overall error for this transducer even at $200^{\circ}$ was less than $3 \%$.

LEF5LHDU: The LEF5LHDU strain gage shows poor temperature compensation. It is possible, though, that the results would be different had all forces been run at all temperatures. The interpretation of the LEF5LHDU temperature data remains ambiguous.

The pressure transducers displayed two distinct peaks, but neither peak was centered at more than $1 \%$ error, and both peaks showed very low scatter. 


\section{FINITE ELEMENT CORRELATION}

FEA models were built for the LEF2LSO (Figure 12) and LEF5LHDU (Figure 11) hydraulic lines. One of the purposes of FEA was to provide a separate benchmark from which to evaluate the experimental results of the strain gage calibrations. However, it was also hoped that once results from FEA were validated with respect to the experimental results and theoretical calculations, new test conditions that were not undertaken with the actual lines could be "virtually" tested through FEA.

FEA information: Pro/E Version 20 was used to construct the CAD models of the hydraulic lines. These models were then exported to ANSYS 5.5 via IGES files in order to conduct the FEA. Shell elements (SHELL93 and SHELL63) were used to model the geometry, although solid elements (SOLID45) and pipe elements (PIPE16 and PIPE18) were also evaluated. The slight gain in accuracy achieved with solid elements was coupled with a large increase in analysis run time. Pipe elements allowed hoop stress to be more directly measured and had quick run times, but they did not allow any static loads to be applied. Shell elements provided the most flexibility along with reasonable run times, so they were utilized. As noted in figures 11 and 12, fixed constraints were imposed at the ends of both lines. Elements at the strain gage locations were isolated in order to read hoop stresses at that location.

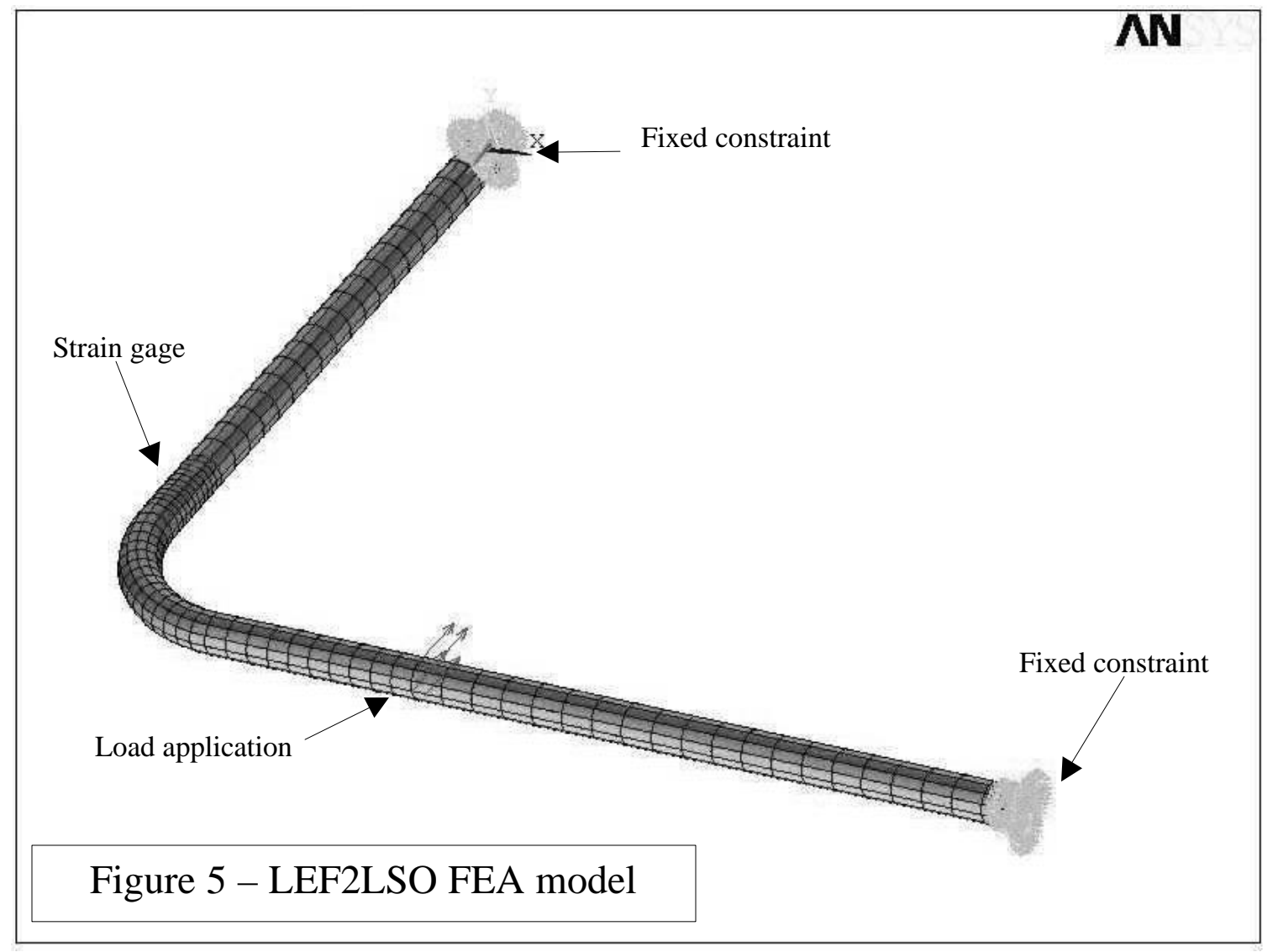

Loading conditions: Internal pressure loads identical to those carried out in the experimental calibrations were imposed on the FEA model, yielding load cases of 500, 1000, 1500, and 2000 psi. Runs with temperature differences were conducted on the LEF2LSO model, yielding four more load 
cases at 0, 32, 150, and 200 degrees Fahrenheit. However, the difference in hoop stress due to temperature was negligible, so FEA temperature runs were not conducted for LEF5LHDU. This is as expected, as the lines should primarily respond to temperature in the axial, not radial direction. Hoop stress is measured tangent to the outside diameter of the line due to radial expansion or contraction, so there should only be minute changes in hoop stress due to temperature. In addition, since the strain gages were to be temperature-compensated, in theory there should be no change in hoop stress (although as mentioned the temperature compensation on the LEF5LHDU line is questionable). As in the experimental calibrations, four different static loads were applied to each line, although as noted the compressive load on LEF2LSO is unknown as load cells were not part of the experimental setup until after that calibration was completed. Combinations of pressure and static load cases were combined to yield FEA data that could be compared to the experimental data. In addition, torque loads in the clockwise $(\mathrm{CW})$ and counter-clockwise $(\mathrm{CCW})$ directions were applied to the LEF2LSO model to evaluate their effect. The resulting hoop stress change was negligible in the $\mathrm{CW}$ direction but a significant increase in stress was observed in the CCW application of torque. Data from the FEA runs can be found in Appendix B.

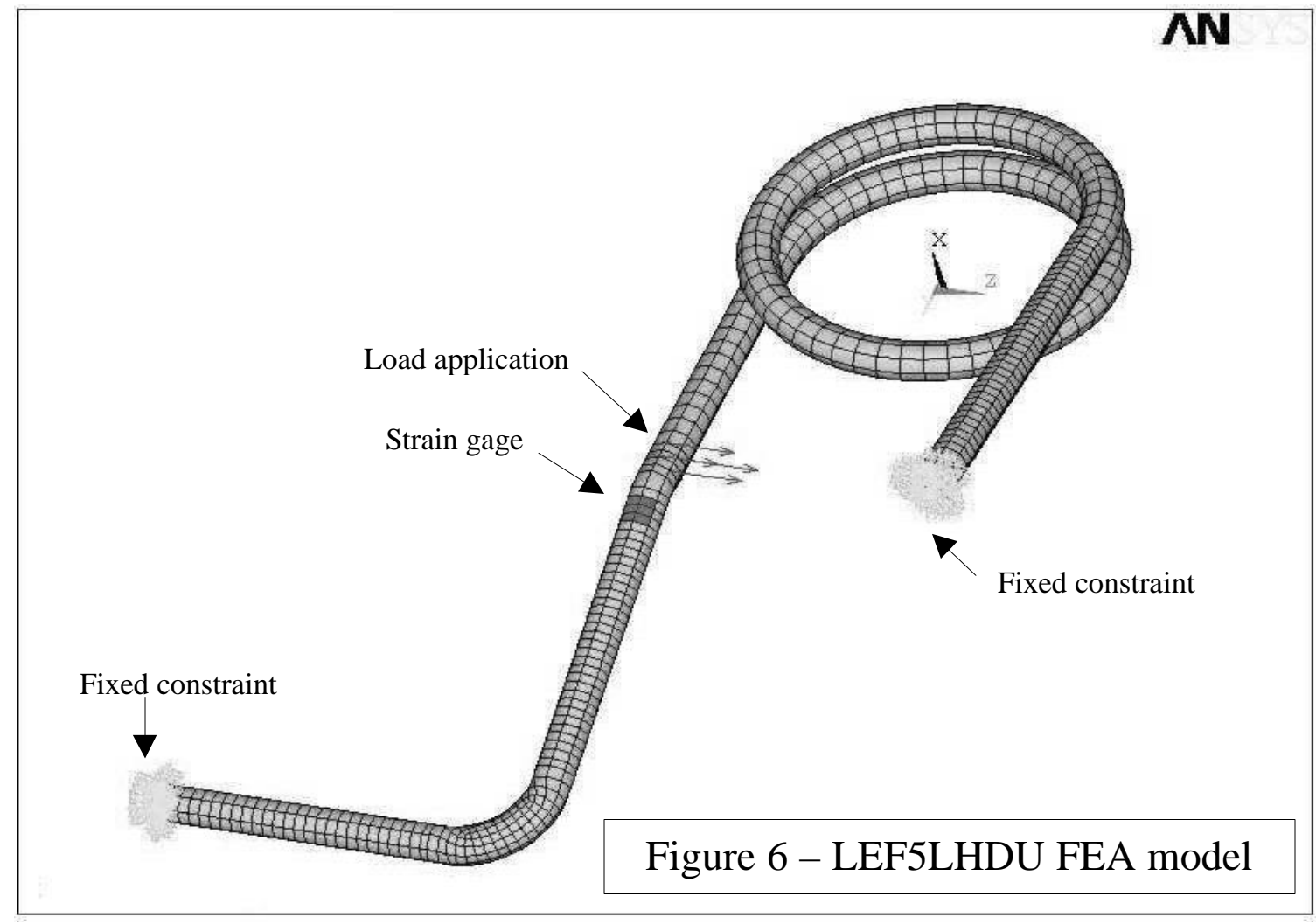

Theoretical error: In order to validate FEA results, stresses were compared to theoretical calculations as well as experimental values (see Appendix B for theoretical calculations). Unfortunately, theoretical calculations were only feasible for hoop stress calculations under internal pressure, so theoretical error calculations for the static loading cases were unavailable. Using theoretical calculations as "truth" values, errors encountered when comparing FEA results to theoretical results were relatively low $4.53 \%$ for LEF2LSO and $.83 \%$ for LEF5LHDU. These error values do not take into account the results at the lowest pressure level, $500 \mathrm{psi}$, as it seems that the FEA model overpredicts the stiffness of the 
Titanium material of the line at such a low pressure, causing high error due to a negative offset. However, above that pressure level, theoretical error levels were consistently low as just mentioned.

Experimental error: Comparing FEA results to experimental results yielded higher error. Using experimental results as "truth" values, average experimental error in the LEF2LSO line was 10.89\%, again discounting errors due to the tendency of the FEA model to overpredict line stiffness. Average experimental error for the LEF5LHDU model was high, 79.16\%, also discounting the 500 psi anomaly in the FEA model. Stresses in the LEF5LHDU FEA model were consistently much higher than in the calibrations, again with the exception of the 500 psi. One source of error for the LEF5LHDU model is the technique in which force was applied to the experimental calibration. The model was loaded at a point very close to the physical location of the strain gage. The resulting stresses in the FEA model had very high gradients in the vicinity of the load application, causing precise readings of stress in the area where the strain gage was located to be somewhat difficult. Another possible explanation for this large error is a discrepancy in the installation procedure of the strain gage. The gages were to be wired in such a manner as to nullify any stresses due to bending in the line; both lines were wired in this manner. In addition, they were to be oriented at an angle (dependant on the material of the line) relative to the hydraulic line axis in order to compensate for the Poisson effect (the tendency of the line to deform in both the radial and axial directions - pressure should only be measured due to radial, not axial, expansion). The strain gages on the LEF2LSO line were installed at this prescribed angle, but the gages on the LEF5LHDU line were instead aligned with the axis of the hydraulic line. It is possible that the small radius of the LEF5LHDU line precluded installing the gages at an angle to the axis. However, the line will still exhibit Poisson effects. Although this definitely affects the measuring of hoop stress on the line, it is believed that this oversight alone is not enough to explain the high errors encountered. Another contributing factor is the poor temperature compensation exhibited by the LEF5LHDU line. This could be a possible indication of other strain gage installation-related errors, since the lack of

temperature compensation is most likely a wiring issue. There are numerous factors involved in the installation of strain gages that can contribute to error; even the most meticulous application typically results in at best approximately 5\% error (Measurement Systems), so the observed discrepancies in installation issues can easily explain some of the errors observed in both lines.

\section{CONCLUSIONS}

The data clearly shows that strain gages respond not only to hoop stress but also to other external forces on hydraulic lines. The uncertainty levels for the two gages tested was approximately $20 \%$, compared to approximately $2.5 \%$ for pressure transducers - the strain gages displayed nearly a full order of magnitude more measurement uncertainty. It is recommended that strain gages be used only when "approximate" data is required, or for trend data. They should not be used for precise measurement of hydraulic line pressures during flight. Safety of flight may be more at risk if precise knowledge of hydraulic line pressure is required for go/no-go decisions. The FEA model conformed closely to the theoretical calculations of hoop stress due to internal pressure only, but in both lines higher errors were encountered when comparing to experimental results. The high disparity between FEA results and LEF5LHDU results is especially obvious, but the multiple procedural errors in gage installation at least partially explain these results. Other dynamic, thermal, and static loading conditions could potentially impose loads on the lines, and may warrant further exploration to determine their impact. 


\section{ACKNOWLEDGEMENTS}

Jim Farrell, Dwaine Fowler, Doug Mullins, Curt Foianini, Tony Cullison, Gary Rumsey, Ted Delbo

\section{REFERENCES}

Dieck, Ronald, Measurement Uncertainty Methods and Applications, Second Edition, Instrument Society of America, Research Triangle Park, NC, 1997.

Abernethy, R.B., and Thompson, Jr., J.W., "Measurement Uncertainty Handbook," Report \# AEDC-TR73-5 / ARO-ETF-TR-72-60, Arnold Engineering Development Center, Arnold Air Force Station, Tennessee, February 1973, Revised January 1980.

Beer, Ferdinand P. and Johnston, E. Russell, Mechanics of Materials, McGraw-Hill Book Company, New York, 1981.

Eagleton, R.H., "Flight Test Aircraft Standard Strain Gage Installation Procedures and Practices Rev D," MDC A0499, McDonnell Douglas Aerospace-East, St. Louis, MO, September 1994.

Measurements Group, Inc., Strain Measuring Systems SC-300 Class Notes, Measurements Group, Inc., Raleigh, NC, 1997.

Young, Warren C., Roark's Formulas for Stress \& Strain, Sixth Edition, McGraw-Hill Book Company, New York, 1989. 